\title{
Intraperitoneal Paclitaxel Is Useful as Adjuvant Chemotherapy for Advanced Gastric Cancer with Serosal Exposure
}

\author{
Joji Kitayama Hironori Ishigami Hironori Yamaguchi \\ Shigenobu Emoto Toshiaki Watanabe \\ Department of Surgical Oncology, University of Tokyo, Tokyo, Japan
}

\section{Key Words}

Peritoneal recurrence $\cdot$ Nodal recurrence $\cdot$ Gastric cancer $\cdot$ Serosal invasion $\cdot$ Intraperitoneal chemotherapy · Paclitaxel

\begin{abstract}
Background: Intraperitoneal administration of paclitaxel (PTX) can elicit a marked clinical response in peritoneal metastases of gastric cancer. Methods: In this study, we retrospectively analyzed the clinical outcome of 17 patients who underwent R0 resection with D2 dissection for advanced gastric cancer with macroscopic serosal exposure and received intraperitoneal PTX as adjuvant therapy. Results: A pathological study revealed that the depth of invasion of the primary tumor was pT4a or pT4b in 10 cases, and that the pN stage was more than pN2 in 8 cases. Genetic analysis of peritoneal lavage fluid was performed in 14 cases, all of which were positive for carcinoembryonic antigen mRNA. In these patients, PTX was intraperitoneally administered at $20-60 \mathrm{mg} / \mathrm{m}^{2}$ with oral S-1 for 3-36 months after surgery. In a median follow-up period of 66 months, recurrence occurred in the liver and peritoneum in $2(11.7 \%)$ and $1(5.9 \%)$ patients, respectively, and no nodal recurrence was observed. Five-year overall survival and disease-free survival were 88.2 and $82.3 \%$, respectively. Conclusion: Since these patients are considered to be a high-risk group for peritoneal recurrence, this result strongly suggests that adjuvant chemotherapy including intraperitoneal PTX is a promising protocol to improve the outcome of patients with advanced gastric cancer with serosal exposure.




\section{Introduction}

Gastric cancer is the fourth leading cause of cancer-related deaths worldwide, and peritoneal dissemination is the most life-threatening form of metastasis and recurrence in patients with advanced gastric cancer [1]. In spite of the recent improvement in chemotherapeutic treatment for solid cancers, the effect of systemic chemotherapy on peritoneal metastases is still limited, possibly because of the peritoneum-plasma barrier which prevents effective drug delivery from the systemic circulation into the peritoneal cavity [2].

In contrast, intraperitoneal administration of paclitaxel (PTX) was developed to enhance antitumor activity against peritoneal metastases by maintaining a high concentration of the drug in the peritoneal cavity over a long period, and its clinical effects have been verified by a number of convincing clinical trials on ovarian cancer with peritoneal metastases $[3,4]$. These results inspired us to use intraperitoneal PTX for peritoneal metastases of gastric cancer, and we therefore designed a study involving intraperitoneal and intravenous PTX combined with oral S-1 chemotherapy. A phase I study determined the optimum dose of intraperitoneal PTX to be $20 \mathrm{mg} / \mathrm{m}^{2}$ [5]. We then conducted a phase II study in 40 patients with gastric cancer who had peritoneal metastases, showing a 1-year overall survival rate of $78 \%$ and a median survival time of 23.6 months [6]. In fact, we found that many metastatic nodules had macroscopically disappeared after several courses of the regimen even in cases with malignant ascites [7].

These results suggest that intraperitoneal PTX can be used as an adjuvant therapy to suppress peritoneal recurrence in advanced gastric cancer. According to the results of the Adjuvant Chemotherapy Trial of S-1 for Gastric Cancer (ACTS-GC) [8], postoperative administration of S-1 for 1 year is now considered to be the standard adjuvant treatment for curatively resected stage II/III gastric cancer in Japan. However, 5-year survival data indicate that the benefit of S-1 is less significant in stage III cancer, especially in patients with T4 tumor (serosal exposure) [9]. More recently, the Capecitabine and Oxaliplatin Adjuvant Study in Stomach Cancer (CLASSIC) trial has been performed in Korea, which demonstrates significant benefit in 3-year disease-free survival in stage III gastric cancer [10]. However, even in this study, the effect on peritoneal recurrence was not as marked as that on recurrence at other sites. Therefore, the development of a highly effective adjuvant method to suppress peritoneal recurrence is considered to be the key factor to improve the survival of patients with stage III gastric cancer. In this study, we, therefore, retrospectively examined the clinical course of patients who received intraperitoneal PTX after curative gastrectomy with D2 dissection in our Department, and discussed the possibility of usage of intraperitoneal PTX in an adjuvant setting.

\section{Patients and Methods}

This retrospective study included 17 patients with advanced gastric cancer who received curative gastrectomy with D2 nodal dissection and subsequent adjuvant therapy including intraperitoneal administration of PTX using a subcutaneously placed peritoneal access port from December 2005 to November 2011 in the Department of Surgical Oncology, University of Tokyo, Japan. In all cases, the primary tumor was macroscopically exposed to the serosal surface, and no tumor cells were detected on peritoneal cytological examination (CY0). Surgical specimens were pathologically examined, and all cases were confirmed to be R0 resection. The pathological stage was determined according to the 14th edition of the general rules for gastric cancer published by the Japanese Gastric Cancer Association [11]. In 
Kitayama et al.: Intraperitoneal Paclitaxel Is Useful as Adjuvant Chemotherapy for Advanced Gastric Cancer with Serosal Exposure

14 cases, carcinoembryonic antigen (CEA) mRNA in peritoneal lavage fluid was additionally examined by RT-PCR as described previously [12].

In the initial 11 patients, $20-60 \mathrm{mg} / \mathrm{m}^{2}$ PTX was administered only through the intraperitoneal route for 6-36 months after surgery. Ten of these patients received oral S-1 (40$80 \mathrm{mg} /$ day) for 2-14 months. In the other 6 cases, patients received a fixed protocol of intraperitoneal $\left(20 \mathrm{mg} / \mathrm{m}^{2}\right)$ and intravenous $\left(50 \mathrm{mg} / \mathrm{m}^{2}\right)$ PTX and S-1 (80 mg/day), as used for the phase II study for patients with peritoneal metastases [6], for 4-24 months. All the patients were basically treated in the outpatient clinic and followed up for more than 5 years, except the last patient with a 2-year follow-up. Tumor marker levels of CEA, CA19-9, CA125 and CA72-4 were periodically examined every 3-6 months. The patients also received CT examination every 6 months for 2 years after surgery, and every year thereafter until 5 years after surgery, and the images were checked for the presence of recurrence by both radiologists and surgeons.

Disease-free survival was defined as the period between surgery and first recurrence, regardless of where it appeared. Data for patients without events were censored as the date of the final observation, and overall survival and disease-free survival curves were calculated using the Kaplan-Meier method (fig. 1).

\section{Results}

The characteristics of the 17 patients are summarized in table 1 . The primary tumor was restricted to the lower and upper stomach in 7 and 1 cases, respectively, while it was located in more than 2 regions in the other 9 cases, and was macroscopically defined as type 2, 3 and 4 in 1, 9 and 7 cases, respectively. Histologically, undifferentiated carcinoma was predominant in 13 cases, and was partially observed at the invasive front in other 2 cases. The depth of invasion was pathologically defined as pT2 and pT3 in 1 and 6 cases and as pT4a or pT4b in the other 10 cases. Nodal metastases were observed in 14 cases and determined as pN1, pN2, pN3a, and pN3b in 4, 2, 3 and 5 cases, respectively. Thus, 7 and 10 cases were classified as p stage II and III, respectively. It is notable that CEA mRNA was positive in all 14 cases examined, suggesting that the patients were in the high-risk group for peritoneal recurrence.

The detailed treatment methods and outcome are summarized in table 2. Most of the patients received S-1 as standard adjuvant treatment, except 1 patient because of gastrointestinal toxicity. In addition, PTX was given through a peritoneal access port at a dose of 20$60 \mathrm{mg} / \mathrm{m}^{2}$ for 3-36 months after surgery. PTX was administered only by the intraperitoneal route in 11 patients, and together with the intravenous route in the other 6 patients. Recurrence was observed in the liver in 2 patients (11.7\%) at 11 and 24 months, and they died 14 and 34 months after surgery, respectively. Both patients were classified as p stage III, and histological examination of the primary tumor showed predominantly differentiated carcinoma. Peritoneal recurrence was observed only in 1 patient (5.9\%), just before the 5year examination after surgery, who is currently under treatment with a second-line regimen. More impressively, nodal recurrence was observed in none of the patients. In the median follow-up period of 66 months, 5-year overall survival and disease-free survival were calculated as 88.2 and $82.3 \%$, respectively. 


\section{Discussion}

PTX is water insoluble and, for clinical use, conventionally solubilized in a polyoxyethylated castor oil named Cremophor EL and ethanol (i.e., Taxol ${ }^{\circledR}$ ). Due to its large particle size (10-12 nm diameter) and hydrophobicity, intraperitoneally administered PTX is slowly absorbed from the peritoneal cavity, which results in prolonged retention in the peritoneal cavity and allows direct penetration of PTX into peritoneal tumors [13]. From its pharmacokinetic characteristics, PTX is considered to be an ideal drug for intraperitoneal chemotherapy. In fact, we have established combination chemotherapy using intraperitoneal PTX together with intravenous PTX and oral S-1, and reported marked clinical effects in gastric cancer with macroscopic peritoneal metastases $[6,7]$. Moreover, Imano et al. [14] have demonstrated that cancer cells were totally eradicated from intra-abdominal fluid at $24 \mathrm{~h}$ after intraperitoneal PTX.

From these clinical results, it is expected that regimens including intraperitoneal PTX could be a powerful method to suppress peritoneal recurrence in selected patients with advanced gastric cancer who have undergone surgical resection of the primary tumor. With this concept, we used intraperitoneal PTX in an adjuvant setting for 17 patients with gastric cancer with macroscopic serosal exposure, and found that peritoneal recurrence was observed in only 1 case (5.8\%) during the median follow-up period of more than 5 years. According to the 5-year ACTS-GC results, peritoneal recurrence was reported to occur in 18.9 and $14.6 \%$ in the surgery alone and S-1 group, respectively [9]. In the 3-year results of the CLASSIC trial, peritoneal recurrence was observed in 56 of 515 patients (10.8\%) with surgery alone and 47 of 520 patients (9.0\%) who received XELOX therapy [10]. Since the patients in our series had a primary tumor with macroscopic serosal exposure and were thus associated with much higher risk for peritoneal recurrence as compared with patients in these two adjuvant studies, this result, although the number of the cases is not sufficient, suggests great potential for intraperitoneal PTX to suppress recurrence in the peritoneum.

Two patients with a p stage III tumor, however, developed recurrence in the liver. Both patients had a primary tumor consisting mainly of a differentiated type carcinoma with a relatively high potential to metastasize to the liver. Since PTX in the abdominal cavity is not easily transferred to the systemic circulation, it may be reasonable that intraperitoneal PTX is less effective in distant organs and thus stronger regimens may be necessary to reduce hematogenous recurrence in those cases.

However, another interesting finding is that nodal recurrence was never detected in these 17 patients, although most of the patients showed a highly advanced pN stage. Since none of the patients received extended nodal lymphadenectomy, this result is totally dependent on the pharmacological effects. Previous studies have shown that PTX retained in the abdominal cavity is mostly absorbed through the lymphatic system [13]. In fact, we confirmed in an animal study that the concentration of PTX in mesenteric lymph nodes was maintained at a higher level after intraperitoneal than after intravenous administration [15]. Thus, in our patients, it is speculated that a large amount of PTX was delivered to regional lymph nodes after intraperitoneal administration, where PTX might effectively suppress the growth of micrometastases or isolated tumor cells.

In recent clinical trials, the efficacy of intravenous PTX as an adjuvant drug has been evaluated for advanced gastric cancer. However, our data suggest that intraperitoneal PTX can produce more marked effects to suppress peritoneal as well as nodal recurrence in advanced gastric cancer with a high risk of recurrence in those sites. The result appears to be reasonable from the pharmacokinetic point of view. In adjuvant treatment, it is critically important to deliver the drugs preferentially to sites that are most liable to develop 
recurrence. Although the retrospective result with a small sample size has its limitation and a clinical study is essential to see the real feasibility, it raises the possibility that adjuvant chemotherapy including intraperitoneal PTX could be a promising strategy to improve the outcome of patients with advanced gastric cancer, especially those with serosal exposure.

\section{Acknowledgements}

We thank Ms. Ikuko Nieda for her clerical work. This study was funded by the Ministry of Health, Labor and Welfare of Japan.

\section{References}

1 Jemal A, Bray F, Center MM, et al: Global cancer statistics. CA Cancer J Clin 2011;61:69-90.

-2 Jacquet P, Sugarbaker PH: Peritoneal-plasma barrier. Cancer Treat Res 1996;82:53-63.

-3 Markman M, Brady MF, Spirtos NM, et al: Phase II trial of intraperitoneal paclitaxel in carcinoma of the ovary, tube, and peritoneum: a Gynecologic Oncology Group Study. J Clin Oncol 1998;16:2620-2624.

4 Armstrong DK, Bundy B, Wenzel L, et al: Intraperitoneal cisplatin and paclitaxel in ovarian cancer. N Engl J Med 2006;354:34-43.

5 Ishigami H, Kitayama J, Otani K, et al: Phase I pharmacokinetic study of weekly intravenous and intraperitoneal paclitaxel combined with S-1 for advanced gastric cancer. Oncology 2009;76:311-314.

-6 Ishigami H, Kitayama J, Kaisaki S, et al: Phase II study of weekly intravenous and intraperitoneal paclitaxel combined with S-1 for advanced gastric cancer with peritoneal metastasis. Ann Oncol 2010;21:67-70.

-7 Kitayama J, Ishigami H, Yamaguchi H, et al: Salvage gastrectomy after intravenous and intraperitoneal paclitaxel (PTX) administration with oral S-1 for peritoneal dissemination of advanced gastric cancer with malignant ascites. Ann Surg Oncol 2013, Epub ahead of print.

8 Sakuramoto S, Sasako M, Yamaguchi T, et al: Adjuvant chemotherapy for gastric cancer with S-1, an oral fluoropyrimidine. N Engl J Med 2007;357:1810-1820.

$>9$ Sasako M, Sakuramoto S, Katai H, et al: Five-year outcomes of a randomized phase III trial comparing adjuvant chemotherapy with S-1 versus surgery alone in stage II or III gastric cancer. J Clin Oncol 2011;29:4387-4393.

10 Bang YJ, Kim YW, Yang HK, et al: Adjuvant capecitabine and oxaliplatin for gastric cancer after D2 gastrectomy (CLASSIC): a phase 3 open-label, randomised controlled trial. Lancet 2012;379:315-321.

-11 Japanese Gastric Cancer Association: Japanese classification of gastric carcinoma - 2nd English edition. Gastric Cancer 1998;1:10-24.

12 Kodera Y, Nakanishi H, Ito S, et al: Quantitative detection of disseminated free cancer cells in peritoneal washes with real-time reverse transcriptase-polymerase chain reaction: a sensitive predictor of outcome for patients with gastric carcinoma. Ann Surg 2002;235:499-506.

13 Markman M: Intraperitoneal antineoplastic drug delivery: rationale and results. Lancet Oncol 2003;4:277283.

14 Imano M, Imamoto H, Itoh T, et al: Impact of intraperitoneal chemotherapy after gastrectomy with positive cytological findings in peritoneal washings. Eur Surg Res 2011;47:254-259.

$\checkmark 15$ Soma D, Kitayama J, Ishigami H, et al: Different tissue distribution of paclitaxel with intravenous and intraperitoneal administration. J Surg Res 2009;155:142-146. 
Kitayama et al.: Intraperitoneal Paclitaxel Is Useful as Adjuvant Chemotherapy for Advanced Gastric Cancer with Serosal Exposure

Table 1. Clinicopathological findings of the 17 patients who received intraperitoneal PTX in an adjuvant setting

\begin{tabular}{|c|c|c|c|c|c|c|c|c|c|c|}
\hline $\begin{array}{l}\text { Patient } \\
\text { No. }\end{array}$ & $\begin{array}{l}\text { Age, } \\
\text { gender }\end{array}$ & Surgery & Locus & $\begin{array}{l}\text { Macroscopic } \\
\text { appearance }\end{array}$ & Histology & pT & $\mathrm{pN}$ & mLN & p stage & $\begin{array}{l}\text { CEA } \\
\text { mRNA }\end{array}$ \\
\hline 1 & $77, \mathrm{~F}$ & DG & $\mathrm{L}$ & 3 & muc $>$ tub & $\mathrm{T} 4 \mathrm{a}$ & $\mathrm{N} 2$ & $5 / 49$ & IIIB & + \\
\hline 2 & $63, \mathrm{~F}$ & $\mathrm{TG}+\mathrm{S}$ & MU & 3 & muc $>$ pap & $\mathrm{T} 4 \mathrm{a}$ & N3b & $38 / 56$ & IIIC & + \\
\hline 3 & $47, \mathrm{~F}$ & DG & $\mathrm{L}$ & 4 & sig & $\mathrm{T} 2$ & N1 & $1 / 49$ & IIA & ND \\
\hline 4 & $60, \mathrm{M}$ & DG & $\mathrm{L}$ & 3 & tub & T3 & N1 & $1 / 70$ & IIB & ND \\
\hline 5 & $28, F$ & $\mathrm{TG}+\mathrm{S}$ & UM & 4 & por2>sig & T3 & N3b & $16 / 81$ & IIIB & + \\
\hline 6 & $76, \mathrm{M}$ & DG & LM & 3 & por $2>$ tub & $\mathrm{T} 3$ & No & $0 / 41$ & IIA & + \\
\hline 7 & $57, \mathrm{M}$ & $\mathrm{TG}+\mathrm{S}$ & MUL & 4 & tub>por1 & $\mathrm{T} 4 \mathrm{a}$ & $\mathrm{N} 3 \mathrm{~b}$ & $26 / 32$ & IIIC & + \\
\hline 8 & $69, \mathrm{M}$ & $\mathrm{TG}+\mathrm{TC}$ & UE & 3 & por & $\mathrm{T} 4 \mathrm{~b}$ & N3b & $18 / 62$ & IIIC & + \\
\hline 9 & $69, \mathrm{M}$ & DG & LM & 3 & muc $>>$ sig & T3 & N1 & $1 / 45$ & IIB & + \\
\hline 10 & $70, \mathrm{M}$ & DG & $\mathrm{L}$ & 3 & tub>por1 & $\mathrm{T} 4 \mathrm{a}$ & No & $0 / 25$ & IIB & + \\
\hline 11 & $73, \mathrm{M}$ & DG & $\mathrm{L}$ & 2 & por & $\mathrm{T} 4 \mathrm{a}$ & $\mathrm{N} 2$ & $4 / 36$ & IIIB & + \\
\hline 12 & $57, \mathrm{M}$ & DG & LD & 3 & pap/tub & $\mathrm{T} 3$ & N3a & $8 / 67$ & IIIB & + \\
\hline 13 & $57, \mathrm{M}$ & DG & $\mathrm{L}$ & 3 & por & $\mathrm{T} 3$ & N1 & $2 / 19$ & IIB & + \\
\hline 14 & $48, \mathrm{M}$ & DG & $\mathrm{L}$ & 4 & muc $>$ sig & $\mathrm{T} 4 \mathrm{a}$ & No & $0 / 61$ & IIB & + \\
\hline 15 & $63, \mathrm{M}$ & $\mathrm{TG}+\mathrm{S}$ & MUL & 4 & por & $\mathrm{T} 4 \mathrm{a}$ & N3a & $8 / 67$ & IIIC & + \\
\hline 16 & $55, \mathrm{M}$ & $\mathrm{TG}$ & LUM & 4 & por & $\mathrm{T} 4 \mathrm{a}$ & N3a & $7 / 26$ & IIIC & + \\
\hline 17 & $43, \mathrm{M}$ & $\mathrm{TG}+\mathrm{S}$ & LUM & 4 & por & $\mathrm{T} 4 \mathrm{a}$ & N3b & $8 / 35$ & IIIC & ND \\
\hline
\end{tabular}

$\mathrm{DG}=$ Distal gastrectomy; $\mathrm{TG}=$ total gastrectomy; $\mathrm{S}=$ splenectomy; $\mathrm{TC}=$ total colectomy; $\mathrm{U}=$ upper; $\mathrm{M}$ = middle; $\mathrm{L}=$ lower; $\mathrm{UE}=$ upper extremity; $\mathrm{mLN}$ = number of metastatic lymph nodes of total lymph nodes resected; $\mathrm{ND}=$ not determined. $\mathrm{pT}$, $\mathrm{pN}$, and p stage was determined in 17 patients. CEA mRNA in peritoneal lavages was determined by RT-PCR. 
Kitayama et al.: Intraperitoneal Paclitaxel Is Useful as Adjuvant Chemotherapy for Advanced Gastric Cancer with Serosal Exposure

Table 2. Treatment method and outcome of the 17 patients who received intraperitoneal PTX in an adjuvant setting

\begin{tabular}{rllllllll}
\hline $\begin{array}{l}\text { Patient } \\
\text { No. }\end{array}$ & $\begin{array}{l}\text { PTX IP, } \\
\mathrm{mg} / \mathrm{m}^{2}\end{array}$ & $\begin{array}{l}\text { PTX IV, } \\
\mathrm{mg} / \mathrm{m}^{2}\end{array}$ & $\begin{array}{l}\text { Cycle of PTX, } \\
\text { n/weeks }\end{array}$ & $\begin{array}{l}\text { Period of PTX, } \\
\text { months }\end{array}$ & $\begin{array}{l}\mathrm{S}-1, \\
\mathrm{mg} / \text { day }\end{array}$ & $\begin{array}{l}\text { Period of S-1, Outcome } \\
\text { months }\end{array}$ & $\begin{array}{l}\text { Recurrence } \\
\text { site }\end{array}$ \\
\hline 1 & 20 & - & $1 / 2$ & 6 & 40 & 6 & 7-year survival & - \\
2 & 20 & - & $2 / 3$ & 10 & 50 & 2 & 7-year survival & - \\
3 & 20 & - & $1 / 2$ & 5 & 50 & 6 & 7-year survival & - \\
4 & 60 & - & $1 / 2$ & 6 & - & 6 & 7-year survival & - \\
5 & 60 & - & $1 / 2$ & 36 & 40 & 36 & 7-year survival & - \\
6 & 20 & - & $2 / 3$ & 4 & 80 & 12 & 7-year survival & - \\
7 & 60 & - & $2 / 3$ & 14 & 80 & 14 & 34 months dead & liver \\
8 & 50 & - & $1 / 2$ & 3 & 40 & 12 & 7-year survival & - \\
9 & 60 & - & $1 / 2$ & 6 & 40 & 2 & 6-year survival & - \\
10 & 20 & - & $2 / 3$ & 6 & 80 & 6 & 6-year survival & - \\
11 & 20 & - & $1 / 2$ & 9 & 40 & 12 & 6-year survival & - \\
12 & 20 & 50 & $2 / 3$ & 4 & 80 & 6 & 14 months dead & liver \\
13 & 20 & 50 & $2 / 3$ & 6 & 80 & 12 & 5-year survival & - \\
14 & 20 & 50 & $2 / 3$ & 4 & 80 & 12 & 5-year survival & peritoneum \\
15 & 20 & 50 & $2 / 3$ & 24 & 80 & 24 & 5-year survival & - \\
16 & 20 & 50 & $2 / 3$ & 4 & 80 & 12 & 5-year survival & - \\
17 & 20 & 50 & $2 / 3$ & 4 & 80 & 6 & 2-year survival & - \\
\hline
\end{tabular}

IP = Intraperitoneal; IV = intravenous.


Fig. 1. Overall survival (left) and disease-free survival (right) in 17 patients. 